\title{
Exploring the Drivers of Vaccine Hesitancy Toward Childhood and Adolescent Vaccination in Malawi: A Qualitative Study
}

\author{
University of Erfurt \\ Cornelia Betsch \\ University of Erfurt \\ Abdu A. Adamu \\ Cochrane South Africa \\ Khadijah Sanusi Gumbi \\ Ahmadu Bello University \\ Michael G Head \\ University of Southampton \\ Aristide Aplogan \\ Agence de Médecine Préventive Afrique \\ Haoua Tall \\ Agence de Médecine Préventive Afrique \\ Tene-Alima Essoh \\ Agence de Médecine Préventive Afrique
}

Gbadebo Collins Adeyanju ( $\square$ gbadebo.adeyanju@uni-erfurt.de)

Research Article

Keywords: Vaccine Hesitancy, Routine Immunisation, Human Papillomavirus, HPV, Vaccination, Childhood, Adolescent, Behaviour, Malawi, COVID-19

Posted Date: April 15th, 2021

DOI: https://doi.org/10.21203/rs.3.rs-383265/v1

License: (c) (i) This work is licensed under a Creative Commons Attribution 4.0 International License. Read Full License 


\section{Abstract}

Background: Vaccine hesitancy is the delay or refusal of vaccines despite the availability of vaccination services. Although it is considered an important global health threat, it has only rarely been systematically studied within the African context. In this study, we explored the factors that influence vaccine hesitancy among caregivers of children and adolescent girls that are eligible for routine childhood immunisation and the human papillomavirus (HPV) vaccine in Malawi.

Methods: The study employed a qualitative exploratory research design. In-depth information was obtained from caregivers of children and adolescent girls eligible for routine immunisation (RI) and the HPV vaccine, respectively, as well as national and district-level representatives of the expanded program on immunisation (EPI) and community stakeholders through key informant interviews and focus-group discussions. These participants were purposively selected using predefined criteria A total of 25 key informant interviews and two focus-group discussions with 13 participants were conducted. Data collection took place between April 20 and May 24, 2020. The interviews were audio-recorded, transcribed verbatim, and then analysed using a thematic content analysis approach.

Results: The study shows that most of the vaccine-hesitancy drivers for RI also influenced the newly introduced HPV vaccine. Such drivers were inadequate awareness of the vaccination schedule, rumours and conspiracy theories exacerbated by religious beliefs, inability to translate knowledge and attitudes into actual vaccination behaviour, low literacy levels of caregivers, complacency occasioned by the stress of distance and transportation logistics, and disconnection between the community healthcare system and community leaders, among others.

Conclusion: This study provides deeper insights into the determinants of vaccine hesitancy within the Malawian context. The findings can inform the design of context-specific interventions by decision-makers. While the study provided behavioural insights regarding vaccination behaviour in Malawi, there is need for further quantitative studies that can assess the prevalence of the determinants as well as their causal relationships to vaccine uptake.

\subsection{Background}

The contribution of vaccination to global public health and community wellbeing is one of the greatest success stories of modern medicine (WHO, 2019a). Despite this, an estimated 19.7 million infants under the age of one globally have not received three doses of diphtheria, tetanus, and pertussis (DTP3) vaccines (WHO, 2020a; Gupta, 2015). In 2018, 13.5 million children missed vaccination, and this contributed to about 1.5 million deaths from vaccine-preventable diseases (Vanderslott, Dadonaite, \& Roser, 2019; WHO, 2017; Okwo \& Jean, 2015). In the Sub-Saharan Africa (SSA) region (World Bank Group, n.d.), basic vaccination coverage (DTP3) is 76\%, which is lowest among World Health Organisation (WHO) member regions and lower than the global average of 86\% (WUENIC, 2019a). In some country-specific settings, such as Nigeria, vaccination coverage has been stagnant at 57\% between 2016 and 2018 (WUENIC, 2019a, 2019b; WHO, 2019a). Prior to this study, vaccination coverage across the region has been a stalemate (70\%), and even on a declining course in some specific settings, where the number of under-five children missing the third dose of DTP3 vaccines was estimated at 7.4 million (Naimoli, 2008; Mihigo, 2017; Lafond et al., 2015).

In Malawi, the overall vaccination coverage for the past three years has been good. Vaccines are available at all levels of the health system. Despite the country achieving a remarkable percentage in routine immunisation (RI) (92\% for DTP3 in 2018), many children still remained unimmunized (GAVI, 2019). For example, measles-vaccine uptake in 2019 was estimated to be $92 \%$ for the first dose but only $75 \%$ for the second dose (WHO, 2020b; Chirwa et al., 2020). There has overall been a good vaccine uptake for newly introduced immunisations such as the rotavirus (91\%) and pneumococcal vaccine (89\%) (Ntenda et al., 2019); however, HPV vaccination showed a drop from the first to the second dose, for example, in the districts of Rumphi (98\% to $88 \%)$ and Zomba (89\% to 76\%) (MOH, 2014, 2019). In general, less than $70 \%$ of eligible children received at least one vaccine dose despite availability; meanwhile, over $90 \%$ of eligible caregivers who are in- and out-patients on nonvaccination visits to hospitals have missed at least one vaccination, while about 60\% missed more (Ogbuanu et al., 2019; Okeibunor et al., 2018). Vaccine hesitancy is discussed as one reason for difficulties in maximizing uptake in Malawi and globally (Betsch et al., 2015; WHO, 2014, 2016-7; Petrelli, 2018; Larson, 2020). Vaccine hesitancy, in fact, has been labelled one of the ten threats to global public health in 2019 (WHO, 2019b).

Vaccine hesitancy is defined as the delay or refusal of vaccines despite the availability of vaccination services (WHO, 2014). It is influenced by many factors, including safety concerns, rumours and conspiracy theories, and fears of adverse events (Betsch et al., 2015, 2018; Msyamboza, 2017; WHO, 2014; Cooper et al., 2018; Dubé, 2013; Larson, 2016). Despite this, much of the evidence around hesitancy has been focused on highincome settings, and little is known about the extent of and reasons for vaccine hesitancy in low- and middle-income settings (Bhopal \& Nielsen, 2020). While vaccine hesitancy is, in many respects, vaccine-, context-, and geography-specific, the drivers can also be different when introducing new vaccines such as against HPV (the cause of cervical cancer) and forthcoming against COVID-19 (Oyo-Ita et al., 2016; Lazarus et al., 2020) compared to routine childhood vaccinations. Exploring these potential differences is crucial, as knowledge about these factors will facilitate the introduction of new vaccines. A recent review of literature in African settings shows that, vaccine hesitancy is driven by a complex set of 
interrelated and interdependent variables such as caregiver-related factors, health systems-related factors, and community context (Adamu et al, 2021).

Since the introduction of the vaccine against HPV through the pilot phase in Malawi in 2013 and the EPI routine program in January 2019 , the uptake fell short of expectations, stagnating at 80\% (Magombo-Mana, 2019; Msyamboza et al., 2017). There is emerging evidence that hesitancy may negatively affect uptake of the HPV vaccine (Msyamboza et al., 2017; Dochez, 2017). This is despite the fact that cervical cancer constitutes the fourth most-common form of cancer globally among women, and SSA countries carry a big burden (IARC, 2018; Bruni et al., 2017). Out of the countries with the highest prevalence of cervical cancer in 2018, more than $90 \%$ were in the African region (Bray et al., 2018; Globocon, 2018; GAVI, 2019). In Malawi, cervical cancer has become a public health crisis (Msyamboza et al., 2017). Malawi has about the highest incidence of cervical cancer in the world and number one among women aged $15-44$ years with a $50 \%$ mortality rate (Campbell et al. 2016; Bruni et al., 2019; Maseko et al., 2015: 2; Fort et al., 2011: 125). As HPV vaccination can effectively prevent cervical cancer, it is of utmost importance to learn about the factors contributing to the omission of that specific vaccine. This can also shed light on more general factors that are relevant for the introduction of new vaccines.

Studies in the SSA region, including reviews of findings in 13 countries (excluding Malawi), show that barriers to HPV vaccine uptake are associated with not just access to health facilities, but perceived risks of receiving the vaccine, safety and effectiveness concerns, low levels of knowledge and awareness (DiAngi, 2011; Francis et al., 2011; Bisi-Onyemaechi et al., 2018; Cunningham, Davison \& Aronson, 2014; Perlman et al., 2014). Preliminary studies done only in some pilot sites have suggested that the drivers summarised above could also be relevant in Malawi, such as lack of education about cervical cancer and availability of the vaccine against it, adverse events, fear, and mistrust (Vermandere et al., 2015, 2016; Wiyeh et al., 2018; Karafillakis et al., 2019).

Similarly, in a study on newly introduced maternal vaccines in Malawi, fear of adverse events, inadequate capacity of the healthcare system, and lack of awareness were considered challenges for acceptance (Flemming et al., 2019). However, because the HPV vaccine is in its early phase of introduction into the national RI program, there is inadequate behavioural insights available that could more comprehensively explain the low demand for HPV vaccines. Also, the studies above have focused only on school-based implementation, not the general population, which this study targeted.

The Government of Malawi Health Sector Strategic Plan II (2017-2022) has set a goal "of ensuring that the people of Malawi attain the highest possible level of health and quality of life" (Malawi HSSP II, 2017). This will be achieved by ensuring universal coverage of basic health care, which is the obligation of the government guaranteed under the republican constitution. One of the key objectives of this plan is to reduce the burden of communicable diseases by rolling out nationwide immunisation programs. Despite the rollout effort, immunisation is being constrained by vaccine hesitancy. In Malawi, there is a paucity of evidence to support the national government's efforts to achieve the above plan using evidence-based strategies; hence, one of the motivations for this study. Therefore, the goal of this study was to understand factors

that drive vaccine hesitancy through the lenses of immunisation stakeholders and community members in Malawi; both for RI in general and the HPV vaccine in particular. The results of the study can support evidence-informed decision-making by Malawi health-system stakeholders in considering local factors mitigating against the uptake of recommended vaccinations.

\subsection{Method}

The National Health Science Research Committee (NHSRC) in Malawi provided ethical approval (\#20/04/2544). Authorization was also obtained from the Malawi Ministry of Health, reference number MED/1/3. Written informed consent was obtained from all subjects. All methods were performed in accordance with the relevant guidelines and regulations.

\subsection{Study Designand Setting}

This study utilized 25 individual Key Informant Interviews (KII) using a semi-structured interview guide and two multi-stakeholder Focus Group Discussions (FGD). The study considered exploring evidence from both purposively selected key informants and the assembly of community members, not only to corroborate evidence but also to explore the dynamics of the relationship between vaccine demand and supply. The FGD provided an in-depth understanding of contextual or social issues, while KII generated knowledge insights into the phenomena under study (Nyumba et al., 2017; Carter \& Beaulieu, 1992; Mountain States Group, 1999). Therefore, using both methods in this exploratory study enhances conceptualization of the phenomena at hand, exploration of the contextual circumstances surrounding such phenomena, and convergence of the central or identical features, which increases the validity of the study findings (Lambert \& Loiselle, 2008).

The study was conducted in the Salima, Lilongwe, Dowa, and Zomba districts of Malawi (Figure 1). The districts were selected based on the following criteria: one district with high vaccine coverage (Lilongwe), one with low vaccine coverage (Dowa), and one each in an urban (Zomba) and rural (Salima) districts where the HPV vaccine had been implemented. The two latter districts were selected not on the basis of coverage but on the rural-urban difference. 
Samples for both KII and FGD were drawn from the districts highlighted in Figure 1. The respondents were purposively selected. For the KII at the central level, we included the National EPI Manager, one EPI social mobilization officer, one NITAG member, one UNICEF representative, and one WHO representative. At each district level, participants included the district EPI manager, one community leader (CL), one community health care worker (HCW), one religious group representative or representatives of civil society organisations (RGR or CSR), and one teacher from a school where an HPV demonstration project was conducted. For the FGD, included participants were stakeholders and caregivers comprising one community leader, one community health care worker, one religious group representative, one representative of civil society organisations, one caregiver whose daughter was eligible for HPV vaccination, one teacher from a school with the HPV demo project, and one caregiver whose child was eligible for RI.

A total sample size of 25 key informants was interviewed, while six discussants in Zomba and seven in Salima participated in the two focusgroup discussions. For the two focus-group discussions, participants were purposively identified and selected from communities where EPI has implemented programs. Participants at the Salima FGD comprised three females and four males, while participants at Zomba FDG comprised three males and three females. The KII lasted 30 minutes on average, while each of the FGD sessions was conducted for an average time of 90 minutes. The interviews and discussions were voice-recorded and transcribed verbatim.

\subsection{Data Collection Tools}

The interview guide comprised the following themes and topics: knowledge of immunisation ("What do you know or think about immunisation?"); knowledge of HPV/cervical cancer ("Do you know or have you heard about cervical cancer and/or the HPV vaccine?"); attitude toward childhood RI and the HPV vaccine ("Do you think childhood RI is an important topic for you and/or your community?... How?" and "What do you think about the HPV vaccine and the target group?...Why do you think that way?"); barriers against childhood RI ("Do you know about vaccine hesitancy and what are the reasons for low childhood immunisation demand?...Please describe why"); barriers against HPV vaccine acceptance ("Do you know about hesitation against HPV vaccine?...If so, what are they, among whom are they, and why?") (Al-Ayed \& Sheik, 2006; Santos et al., 2020; Ogochukwu et al., 2017; Bangura et al., 2020).

\subsection{Data Analysis}

The data were analysed using thematic deductive content analysis (Elo \& Kyngas, 2008) and focused on identifying enablers and barriers against vaccine acceptance for RI and HPV. The recorded interviews and discussions were transcribed verbatim. Main themes and sub-themes were identified after analysing each individual transcript and categorised based on the above themes explored. Transcribed data were coded as follows: the central level was coded C001 and C002, while districts were coded as KII001 and KII002. The FGDs were coded as FDG001and FGD002. The analysis resulted in the development of a thematic index based on the themes explored, such as knowledge, attitude/perception toward vaccination, and general barriers against vaccine acceptance.

The WHO Strategic Advisory Working Group (SAGE) vaccine hesitancy model (WHO, 2014) was used to organise factors driving vaccine hesitancy in Malawi for both childhood RI and HPV vaccines. The model identified three dimensions for organising vaccine-hesitancy determinants such as vaccine/vaccination-specific issues, individual and group influences, and contextual influences. Vaccine/vaccinationspecific issues are factors surrounding concerns about vaccines or vaccination; individual and group influences refer to individual perceptions or individuals' social environments; and contextual influences aim at understanding vaccine hesitancy arising due to historic, socio-cultural, environmental, health system/institutional, economic, or political factors (WHO, 2014).

In the data, trends of recurring patterns were identified that reflected what factors the participants felt most strongly about. These factors were categorised under knowledge of Rl, perception/attitude toward vaccinations, vaccine importance, specific HPV vaccine and cervical cancer knowledge and attitude, and drivers of vaccine hesitancy on RI and HPV. These are important features for understanding vaccination behaviour and demand among caregivers (Musa et al., 2020; Betsch et al., 2018; Angellilo et al., 1999; Dubé et al., 2019; Larson et al., 2013; Adamu et al, 2021).

\subsection{Results}

The resulting factors that influenced acceptance or non-acceptance of RI and HPV vaccines are summarized in Figure 2 below. Each bubble represents the identified vaccine hesitancy drivers in reference to RI (left), to HPV vaccination (right), or both (middle). The figure summarises results from both KII and FGD. The single categories are explained and detailed below.

\subsection{Factors Driving Vaccine Hesitancy toward Routine Immunisation}

\subsubsection{Vaccine/Vaccination-specific Issues}


Competing or Poorly Scheduled Healthcare Services: Participants reported that based on their experiences, "outreach clinics are opened most of the time around 9:00am. Sometimes outreach clinics are missed by caregivers due to other services scheduled at the same time, such as family planning and ante-natal care services" (HCW 2, Salima District). Outreach clinics are usually makeshift medical services stepped down to reach disadvantaged communities or hard-to-reach terrains, to provide essential healthcare services, especially immunisation services. Participants alluded to missed appointments resulting in incomplete immunisation when all the immunisation services were not centralized in one spot or operated on different schedules.

\subsubsection{Individual and Group Influence}

Lack of Confidence: The study participants acknowledged the presence of RI and other types of immunisation services that are being provided in Malawi. However, most participants reported that despite the number of almost five to six healthcare workers providing some of these services at the facility, which was adequate for the setting, the number of adolescent girls and under-fives receiving vaccinations remained low in some districts. A FGD 001 participant alluded to confidence issues: "Many people do not trust immunisation because of stories they hear" (EPI Manager, Zomba District).

Attitude toward Vaccination versus Behaviour: Study participants acknowledged that vaccination is vital to caregivers for protecting their children against vaccine-preventable diseases and agreed that vaccination is a vital topic within their household. However, immunisation was not considered a top priority: "immunisation is very important but there are other equally important things to the family" (Caregiver 2 - Dowa District; FGD002). Further probing indicated the dominance of husbands in household vaccination decision-making as a factor that prevented turning intentions into behaviour. "Well, even though we know the importance of immunisation, our husbands must still agree before we can carry our children to hospital” (Caregiver 1; FGD, Dowa District). "On major market days, attendance is poor because mothers take husbands' farm produce to market, so they miss childhood immunisation" (HCW 1; FGD, Dowa District).

\subsubsection{Contextual Influence}

Inadequate Resources Decrease Motivation for Vaccination Uptake: Most participants reported a lack of resources and medical equipment at village clinics. These necessitated caregivers to sacrifice their personal items for immunisation activities, hence reducing their motivation and willingness to want to continue immunisation. "We use our own resources (e.g., transportation, sanitary items, furniture, etc.) whenever we want to do vaccination activities at the village" (HCW 2, Lilongwe).

Low Literacy Level of Caregivers: The participants also revealed that their caregivers' literacy levels are very low in the communities. This makes the effective health promotion of issues surrounding the importance of vaccination difficult, especially for those without any formal education: "Sometimes some people distribute pamphlets on immunisation, but many of us cannot read" (Caregiver 1, Zomba district).

Distance and Logistics in Accessing Health Centers: The majority of participants described long distances of travel to the clinics, impacting uptake of vaccination: "Lack of easy access to health centers results in lots of missed immunisation schedules" (Caregivers 1, Zomba District). These sentiments were echoed among all caregivers.

Disconnect between Healthcare System and Community Gatekeepers/Leaders: Most participants revealed that essential stakeholders (e.g., community leaders, religious leaders, etc.) were usually not consulted by the Expanded Program on Immunisation (EPI) and Healthcare System managers. "The EPI does not care about our opinion" (CL, Dowa District). This affected not only attitude, but also the turnout for both RI and adolescent girls for the HPV vaccine.

\subsection{Factors Driving Hesitancy toward the Human Papillomavirus Vaccine}

\subsubsection{Vaccine/Vaccination-specific Issues}

Lack of Confidence in Safety and Effectiveness of HPV Vaccine: There were some levels of awareness and even campaigns; however, the communities are not always confident that the HPV vaccine is safe and effective. In many districts, "parents generally, especially fathers, are reluctant to let their eligible daughters receive the HPV vaccine" (HCW 1-2, Lilongwe, Caregivers 1-2, Zomba). "We have heard about the HPV vaccine, but we are not sure about it" (Caregiver $1 ; \mathrm{RL}$, Dowa/Zomba/Lilongwe).

Attitude Toward Vaccination versus Behaviour: More than half of the study participants in all four districts acknowledged that there was knowledge of the HPV vaccine; however, this knowledge has not translated into behaviour. The negative behaviour might be connected to the perception of existing traditional beliefs and cultural practices, which have not changed about vaccination in general and specifically about a vaccine that targets young girls (HPV vaccine). "We have not been convinced why the vaccine targets our girls specifically" (Caregivers 2 , Lilongwe/Dowa/Lilongwe/Zomba). Therefore, high intentions to vaccinate due to knowledge about the HPV vaccine did not affect uptake behaviour. 
Complacency: The caregivers do not believe cervical cancer was prevalent because there are almost no cervical cancer screening opportunities outside the main city centers. "There is little data to support arguments about high HPV prevalence in our area" (CSO, Salima District). This attitude generated low risk perception of HPV, hence complacent behaviour.

Lack of Awareness of Vaccination Schedule: Participants expressed a lack of awareness of the vaccination schedule (dates/timing) as a reason why caregivers missed both routine and HPV vaccinations.

\subsubsection{Individual and Group Influences}

Misconceptions, Rumours, and Conspiracy Theories: The participants reported a misconception that once their daughters get vaccinated against HPV, they become infertile. Other caregivers queried why HPV vaccines target only girls: "Ignorance among community members because of rumours on the HPV vaccine drives vaccine hesitancy...such as the belief that the HPV vaccine will reduce the libido of girls when they become sexually active and make them become reproductively infertile" (EPI Logistician, Dowa/Zomba).

\subsubsection{Contextual Influences}

Religious Beliefs: Participants discussed that there were no widespread traditional or cultural beliefs among Malawian communities that specifically hindered vaccine acceptance. However, there were some specific misconceptions about the vaccine, especially from the Zion and Apostolic faith sects. These groups denied some aspects of modern medicine, including vaccinations, and amplified conspiracy theories surrounding the HPV vaccine, such as that it promotes immoral behaviour and leads to infertility among the recipients. "The HPV vaccine promotes promiscuity and exposes young girls to sex and abortion" (RL, Salima/Zomba).

\subsection{Discussion}

This study has identified some key drivers behind vaccine hesitancy in Malawi, focusing on childhood RI and also the HPV vaccine more specifically. Factors that were identified here as determinants of vaccine hesitancy included a lack of awareness of the vaccination schedule, inability to translate high knowledge and positive attitudes into actual behaviour, lack of trust in the safety and effectiveness of vaccines, complacency, religious beliefs, rumours, and beliefs in conspiracy theories. This study now provides evidence that some missing vaccinations are also related to aspects of refusal or unwillingness to accept a vaccine and not only a lack of access.

The study shows that on the one hand, misinformation, rumours around sterility, and reluctance by senior family members or fathers are factors that keep caregivers from vaccination, while at the same time, evidence around the prevalence of the deadly diseases is lacking, supporting complacent caregiver behaviour. Importantly, however, the study also shows that even correct knowledge and positive attitudes toward vaccination do not reliably turn into actual vaccination behaviour. This matches the finding that other healthcare activities, such as antenatal care and general out-patient services, are prioritized over vaccination appointments when programs appear to "compete" with each other. The data lead to the speculation that male supremacy in household decision-making negatively affects vaccination uptake behaviour. Further research is required to understand this behaviour.

Meanwhile, the majority of participants acknowledged that caregivers typically wish for their children to be immunised against vaccinepreventable diseases and agreed that vaccination is a vital topic within households. This reflected the relatively unstable national vaccine rates for routine childhood immunisations and indicated that while there is clearly some hesitancy in Malawi, it has not yet translated into widespread declines in childhood vaccination uptake. Effective health promotion and education around immunisation are recommended. Negative attitudes or perceptions of the immunisation program can potentially discourage caregivers from continuing vaccination.

The demand for vaccination requires a general perception that vaccines are safe and effective, thereby increasing the feeling of protection from serious illness (Nichter, 1995; Betsch et al., 2018; Adamu et al, 2021). This requires that immunisation campaigns be perceived to be good-quality and have local targets (Streefland et al., 1999). Moreover, given the conservative nature of the setting, fathers or husbands are an important target group and should be given significant priority in educational and advocacy strategies.

Low levels of literacy influence the understanding of public health messaging, and this is most common in caregivers without any formal education who are more likely to miss vaccination clinics (Adedokun et al., 2017). Studies show that insufficient or low literacy is linked to low levels of protective behaviour and can eventually lead to vaccine hesitancy (Biasio, 2017; 2019). Our findings here also reflect previous studies across Africa, suggesting that residents with low adult literacy have lower acceptance of vaccination (Adetokunboh et al., 2015; Adamu et al, 2021). Vaccination messaging should be target-driven. Written messages directed at communities with low literacy levels should be designed using pictures or symbols that are easy to comprehend. In this regard, an overhaul of the immunisation communication system and educational program of the EPI, which has often focused on urban (high literacy area) compared to rural (low literacy area) settings, must be addressed. Future campaigns should fully consider rural and remote settings in the production planning and dissemination of immunisation knowledge or 
information, including consideration for local languages or dialects (Abiiro et al., 2014). This study exposed this disparity, and efforts should be geared toward addressing it.

Healthcare-seeking behaviour is driven by numerous factors; for example, our findings show that distance and logistics were not always a primary determinant of vaccination decisions but that the perceived severity of a particular disease also played a part in driving healthcareseeking behaviour. The decision to forego or miss an immunisation appointment is not determined by circumstances beyond caregivers' control, but by the convenience of doing it vis-a-vis other commitments and value placed on immunisation. Planning health services is therefore also important. Participants here highlighted that they had to, for example, choose between attending ante-natal or immunisation services. Thus, subsequent national immunisation programs should pay attention to how caregivers can prioritize immunisation while at the same time still making use of other health services. Aligning these appointments, combining the presence of services to allow for multiple interventions in one visit, or incentivizing immunisation may help translate caregivers' intentions into actual behaviour. Aligning services may also allow outreach services to visit more communities in hard-to-reach areas, shortening travel time and accessing more of the population.

Communication with the local "gatekeepers" (for example, village elders) is critical when building and strengthening co-operatives (Naimoli, 2008) and can boost local confidence in healthcare services. Also, integrating an SMS reminder system will go a long way in addressing the vaccination schedule problems identified among caregivers (i.e., for those who have mobile devices).

Vaccine hesitancy, particularly for HPV, is prevalent among some Malawian communities regardless of their cultural or religious affiliations. In the Rumphi and Zomba districts, some parents declined consent for their daughters to receive the vaccine, believing the immunisation to be dangerous (Msyamboza et al., 2017: 3). At the heart of some misconceptions about the HPV vaccine is religion, especially Christian groups such as the Zion and Apostolic faith sects, which are among several groups that continue to doubt the safety of vaccines (Ruijs et al., 2011). In the case of the polio eradication program in countries like Afghanistan, Pakistan, and Nigeria, Muslim fundamentalists have sometimes convinced their followers that "it is an American ploy to sterilize the Muslim communities" (Belluz, 2019). It is important for proactive health promotion campaigns to understand and appropriately counter these sentiments. Therefore, efforts of intervention in Malawi must be directed at opinion leaders and gatekeepers, especially community leaders, who wield strong influence to change the narrative.

While the study provided behavioural insights regarding vaccination behaviour in Malawi, there is need for further quantitative studies that can assess the prevalence of the determinants as well as their causal relationship to vaccine uptake.

\subsection{Study Limitations}

The main challenge experienced during this study was the problem of the COVID-19 pandemic, which resulted in unanticipated travel restrictions. This forced the study to substitute two of the originally planned districts (Nsanje and Rumphi) for convenient ones (Salima and Lilongwe) to enable swift and easy access to the data collection sites. Second, interviews at the central level (Lilongwe) took so long to complete because some respondents were COVID-19 essential-services personnel and found it difficult to make time for interviews. Overall, the limitations were minimal, partly because the infection rate in Malawi during the data collection in March/April 2020 was not significant and COVID-19-related restrictions were not yet fully active in most districts.

\subsection{Conclusion}

The evidence presented here, and the lessons learned from the roll-out of new vaccines such as against HPV, can provide a starting point for creating tailored public health messages that are specific to the Malawi population. This has implications both for current levels of vaccine acceptance, but also for the introduction of any new vaccine, such as the vaccine against COVID-19. The study shows that a network of factors determines vaccine hesitancy for RI and HPV, and some of them are interrelated with one another. Strategies developed to address vaccine hesitancy must be multicomponent and wide-ranging. Invariably, the factors that lower the demand for childhood RI are also key to low demand for the HPV vaccine and vice versa. For the introduction of the new COVID-19 vaccines, the following will be especially important: considering the literacy level of the population and allowing the communication campaigns to be sensitive to local settings; ensuring that messaging on safety and vaccine effectiveness are driven by gatekeepers and religious leaders, especially from the most skeptical Christian sects; and dealing with low risk perception and conspiracy theories inspired by rumours and misinformation by using local celebrities or credible community mobilizers. A proactive and coordinated approach to health promotion will be vital in ensuring high levels of acceptance and increased uptake.

\section{Abbreviations}

AMP Afrique - Agence de Médecine Preventive Afrique

CEREB - Center for Empirical Research in Economics and Behavioural Sciences

CL - Community Leaders

Page $7 / 14$ 
CSO - Civil Society Organisation

DPT3 - Third Dose of Diphtheria, Tetanus, and Pertussis Vaccine

EPI - Expanded Program on Immunisation

FGD - Focus Group Discussion

GAVI - Global Alliance for Vaccine and Immunisation

GHP3 - Global Health Population, Poverty, and Policy

HCW - Healthcare Workers

HPV - Human Papilloma Virus

IARC - International Agency for Research on Cancer

KII - Key Informant Interview

NHSRC - National Health Science Research Committee

RI - Routine Immunisation

RL - Religious Leaders

SDG - Sustainable Development Goals

SSA - Sub-Saharan Africa

TRA - Theory of Reasoned Action

UHC - Universal Health Coverage

UNICEF - United Nations Children Emergency Fund

VPD - Vaccine Preventive Disease

WHO - World Health Organisation

WUENIC - WHO UNICEF Immunisation Coverage Estimates

\section{Declarations}

\section{Ethics approval and consent to participate}

Ethical clearance from relevant authorities was obtained. Ethics approval from the National Health Sciences Research Committee (NHSRC) in Malawi was received, with protocol reference number 20/04/2544. Also, authorization was obtained from the Malawi Ministry of Health, reference number MED/1/3. Written informed consent was obtained from all subjects.

\section{Consent for publication}

Not applicable.

\section{Availability of data and materials}

The datasets used and/or analysed during the current study are available from the corresponding author on reasonable request.

\section{Competing interests}

The authors declare that they have no competing interests.

\section{Funding}


This work was supported by an unconditional educational grant from Merck Sharp and Dohme (MSD) through the Agence de Médecine Préventive (AMP) Afrique. However, they have no influence on the content and outcome of the study.

\section{Authors' Contributions}

Conceptualization: GCA, TAE, AAA, HT and AA.

Methodology: GCA, TAE and AAA.

Investigation: GCA, TAE and AAA.

Result Analysis: GCA, TAE, and KSG.

Writing - original draft: GCA, TAE, KSG, MGH and CB.

Writing - review \& editing: GCA, TAE, AAA, MGH and CB.

All authors have read and approved the final manuscript.

\section{ACKNOWLEDGMENTS}

This study acknowledges the support of the Malawi Ministry of Health, the management and staff of the Expanded Program on Immunisation (EPI), Dr. Mike Nenani Chisema (Malawi EPI Country Manager), Temwa Mzengeza (Malawi EPI - National RI Officer), Mr. Luciano Njoka Abraham (Salima District EPI Coordinator), and EPI district stakeholders in the Lilongwe, Salima, Dowa, and Zomba districts. Finally, many thanks to the health facilities used for data collection, such as Salima District Hospital, Khombedza Health Center, Dowa District Hospital, Dzaleka Health Center, Zomba District Hospital, Matawale Health Center, Bwaila Hospital, and the Area 15 Health Center.

\section{References}

1. Adamu, AA., Essoh, TA, Adeyanju, GC., Jalo, RI., Saleh, Y., Aplogan A. \& Wiysonge, CS: Drivers of hesitancy towards recommended childhood vaccines in African settings: a scoping review of literature from Kenya, Malawi and Ethiopia, Expert Review of Vaccines, 2021. DOI: https://doi.org/10.1080/14760584.2021.1899819

2. Abiiro, G. A., Mbera, G. B., \& De Allegri, M. (2014). Gaps in universal health coverage in Malawi: a qualitative study in rural communities. BMC health services research, 14, 234. https://doi.org/10.1186/1472-6963-14-234

3. Adedokun, S., Uthman, O., Adekanmbi, V. T., \& Wiysonge, C. S. (2017). Incomplete childhood immunization in Nigeria: A multilevel analysis of individual and contextual factors. BMC Public Health, 1-10.

4. Adetokunboh, O., Schoonees, A., Balogun, T., \& Wiysonge, CS. (2015). Efficacy and safety of abacavir-containing combination antiretroviral therapy as first-line treatment of HIV infected children and adolescents: A systematic review and meta-analysis. BMC Infectious Diseases, 113.

5. Al-Ayed IH, Sheik S. Knowledge \& practices of childhood immunization among primary health care providers in riyadh city: part ii precautions and contraindications to vaccination. J Family Community Med. 2006;13(1):19-24.

6. Angelillo IF, Ricciardi G, Rossi P, Pantisano P, Langiano E, Pavia M. (1999). Mothers and vaccination: knowledge, attitudes, and behaviour in Italy. Bull World Health Organ; 77(3):224-9. PMID: 10212512; PMCID: PMC2557613.

7. Bangura, J.B., Xiao, S., Qiu, D. et al.Barriers to childhood immunization in sub-Saharan Africa: A systematic review. BMC Public Health. 2020; 20: https://doi.org/10.1186/s12889-020-09169-4

8. Belluz, J. (2019). Religion and vaccine refusal are linked. We have to talk about it. Vox. Retrieved June 2, 2020, from Vox: https://www.vox.com/2019/6/19/18681930/religion-vaccine-refusal

9. Betsch C, Böhm R, Chapman GB. (2015). Using Behavioral Insights to Increase Vaccination Policy Effectiveness. Policy Insights from the Behavioral and Brain Sciences. 2(1):61-73. doi:1177/2372732215600716

10. Betsch, C., Schmid, P., Heinemeier, D., Korn, L., Holtmann, C. \& Böhm, R. (2018). Beyond confidence: Development of a measure assessing the 5C psychological antecedents of vaccination. PLOS one, 13(12). https://doi.org/10.1371/journal.pone.0208601.

11. Bhopal S, Nielsen M. (2020). Vaccine hesitancy in low- and middle-income countries: potential implications for the COVID-19 response. Archives of Disease in Childhood. doi: 10.1136/archdischild-2020-318988

12. Biasio , L. T. (2017). Vaccine hesitancy and health literacy. Human vaccines \& immunotherapeutics, $701-702$.

13. Biasio, L. R. (2019). Vaccine literacy is undervalued. Human Vaccines \& Immunotherapeutics, 2552-2553. 
14. Bisi-Onyemaechi, A.I., Chikani, U.N., Nduagubam, O. (2018). Reducing incidence of cervical cancer: knowledge and attitudes of caregivers in Nigerian city to human papilloma virus vaccination, Infectious Agents and Cancer, 13(29). https://doi.org/10.1186/s13027-018-0202-9

15. Bray , F., Ferlay , J., Soerjomataram , I., Siegel , R. L., Torre, L. A., \& Ahmedin, J. (2018). Global Cancer Statistics 2018: GLOBOCAN estimates of incidence and mortality worldwide for 36 cancers in 185 countries. Cancer Journal for Clinicians, 394-424.

16. Bruni L., Albero G., Serrano B., Mena M., Gómez D., Muñoz J., Bosch FX., De Sanjosé (2017). “Human Papillomavirus and Related Diseases in Africa”. ICO/IARC Information Centre on HPV and Cancer (HPV Information Centre). Summary Report 2019: June 17. Pp. iv.

17. Campbell, C., Kafwafwa, S., Brown, H., Walker, G., Madetsa, B., Deeny, M., Cubie, H. A. (2016). Use of thermo-coagulation as an alternative treatment modality in a 'screen-and- treat' programme of cervical screening in rural Malawi: Use of Thermo-Coagulation in a 'Screen-andTreat' Programme. International Journal of Cancer, 139(4), 908-915. https://doi.org/10.1002/ijc.30101

18. Carter, K.A. \& Beaulieu, L.J. (1992). Conducting A Community Needs Assessment: Primary Data Collection Techniques. Gainesville, FL: University of Florida-Institute of Food and Agricultural Sciences. Retrieved February 3, 2004 from: http://edis.ifas.ufl.edu/pdffiles/HE/HE06000.pdf

19. Chirwa, Geoffrey., Wilkins, Karen Annette., Mercer, David John. (2020). Descriptive study of measles vaccination second dose reporting and barriers to improving coverage in six districts in Malawi. Pan African Medical Journal. Volume 35 (1): 5.

20. Cooper S, Betsch C, Sambala EZ, Mchiza N, Wiysonge CS. (2018). Vaccine hesitancy - a potential threat to the achievements of vaccination programmes in Africa. Hum Vaccin Immunother. 14(10):2355-2357. doi: 10.1080/21645515.2018.1460987.

21. Cunningham, M.S., Davison, C. \& Aronson, K.J. (2014). HPV vaccine acceptability in Africa: A systematic review, Preventive Medicine, 69, 274-279.

22. DiAngi, Y. T., Panozzo, C. A., Ramogola-Masire, D., Steenhoff, A. P., \& Brewer, N. T. (2011). A cross-sectional study of HPV vaccine acceptability in Gaborone, Botswana. PLOS ONE, 6(10), e25481. https://doi.org/10.1371/journal.pone.0025481

23. Dochez, Carine., Burnett, Rosemary J., Mbassi, Symplice Mbola., Were, Fred., Musyoki, , Trovoada, Daisy., Mphahlele, Jeffrey, M. (2017). Improving skills and institutional capacity to strengthen adolescent immunisation programmes and health systems in African countries through HPV vaccine introduction, Papillomavirus Research, Volume 4, Pages 66-71, https://doi.org/10.1016/j.pvr.2017.08.003

24. Dubé, E. L. (2013). Vaccine hesitancy: an overview. Human vaccines \& immunotherapeutics, 1763-1773.

25. Dubé, Ė., Farrands, A., Lemaitre, T., Boulianne, N., Sauvageau, C., Boucher, F. D., Tapiero, B., Quach, C., Ouakki, M., Gosselin, V., Gagnon, D., De Wals, P., Petit, G., Jacques, M. C., \& Gagneur, A. (2019). Overview of knowledge, attitudes, beliefs, vaccine hesitancy and vaccine acceptance among mothers of infants in Quebec, Canada. Human vaccines \& immunotherapeutics, 15(1), 113-120.

https://doi.org/10.1080/21645515.2018.1509647

26. Elo, S \& Kyngas, H. (2008). The qualitative content analysis process. J Adv Nurs, 62, pp. 107-115

27. Fleming JA, Munthali A, Ngwira B, Kadzandira J, Jamili-Phiri M, Ortiz JR, Lambach P, Hombach J, Neuzil KM, Stepanchak M, Bhat N. (2019). Maternal immunization in Malawi: A mixed methods study of community perceptions, programmatic considerations, and recommendations for future planning. Vaccine. 37(32): 4568-4575. doi: 10.1016/j.vaccine.2019.06.020.

28. Fort, V.K., Makin, M.S., Siegler, A.J., Ault, K., Rochat, R. (2011). Barriers to cervical cancer screening in Mulanje, Malawi: A qualitative study. Patient Prefer Adher, 5, 125-131.

29. Francis, S.A., Battle-Fisher, M., Liverpool, J., Hipple, L., Mosavel, M., Soogun, S., Mofammere N. (2011). A qualitative analysis of South African women's knowledge, attitudes, and beliefs about HPV and cervical cancer prevention, vaccine awareness and acceptance, and maternal-child communication about sexual health. Vaccine, 29, 8760-8765.

30. Global Alliance for Vaccine and Immunization (GAVI) (2019). Annual Progress Report: Malawi.

31. New Global Cancer Data: GLOBOCAN 2018. (2018, Sept. 12). Retrieved Dec. 5, 2020. https://www.uicc.org/news/new-global-cancer-dataglobocan-2018.

32. Gupta . P, P. (2015). Determinants of immunization coverage in Lucknow district. N Am J. Med Sci. 36-40.

33. International Agency for Research on Cancer (IARC) 2018 Report, (2018: Sept. 12). World Health Organisation (WHO).

34. Karafillakis E, Simas C, Jarrett C, Verger P, Peretti-Watel P, Dib F, De Angelis S, Takacs J, Ali KA, Pastore Celentano L, Larson H. (2019). HPV vaccination in a context of public mistrust and uncertainty: a systematic literature review of determinants of HPV vaccine hesitancy in Hum Vaccin Immunother. 15(7-8):1615-1627. doi: 10.1080/21645515.2018.1564436.

35. Lafond, A., Kanagat , N., Steinglass , R., Fields , R., Sequeira , J., \& Mookherji , S. (2015). Drivers of routine immunization coverage improvement in Africa: findings fromdistrict-level case studies. Health Policy Plan, 298-308.

36. Lambert SD, Loiselle CG. (2008). Combining individual interviews and focus groups to enhance data richness. J Adv Nurs. 62(2): 228-37. doi: 10.1111/j.1365-2648.2007.04559.x.

37. Larson, H. J. (2016). The State of Vaccine Confidence. Global Insights Through a 67-Country Survey EBioMedicine, 12, $295-301$. 
38. Larson, Heidi et al. (2013). “A Multidisciplinary Research Agenda for Understanding Vaccine-Related Decisions.” Vaccines vol. 1,3 $293-304$. doi:10.3390/vaccines 1030293

39. Larson H. J. (2020). Japan's HPV vaccine crisis: act now to avert cervical cancer cases and deaths. Lancet Public Heal. 5: e184-5

40. Lazarus, J.V., Ratzan, S.C., Palayew, A. et al. (2020). A global survey of potential acceptance of a COVID-19 vaccine. Nat Med. https://doi.org/10.1038/s41591-020-1124-9

41. Magombo-Mana, K. (2019, January 12.) Malawi launches HPV vaccine campaign: Minister Muluzi says cervical cancer leading cause of women's deaths. Nyasa Times. Retrieved Nov. 16, 2020 from: https://www.nyasatimes.com/malawi-launches-hpv-vaccine-campaignminister-muluzi- says-cervical-cancer-leading-cause-of-womens-deaths/

42. Malawi Strategic Health Sector Strategic Plan II (2017-2022), (2017, Jul.). Towards Universal Health Retrieved Nov. 14, 2020 from https://www.healthdatacollaborative.org/fileadmin/uploads/hdc/Documents/Country_documents/HSSP_II_Final_HQ_complete_file.pdf.pdf

43. Malawi Ministry of Health (MOH), (2019). Malawi National Routine Immunization data 2019, unpublished.

44. Malawi Ministry of Health (MOH), (2014), May). HPV Vaccine demonstration project evaluation in Malawi Technical report

45. Maseko, F.C., Chirwa, M.L., Muula, A.S. (2015). Health systems challenges in cervical cancer prevention program in Malawi. Glob Health Action, 8; 26282.

46. Mihigo, R. O. (2017). Challenges of immunization in the African Region. The Pan African medical journal, 27.

47. Mountain States Group, Inc (1999). Community Engagement-Needs Assessment: Conducting Key Informant and Focus Group Interviews. Boise, ID: Mountain States Group. Retrieved February 11, 2004 from: http://deltarhpi.ruralhealth.hrsa.gov/tools2.shtml

48. Msyamboza, K.P., Mwagomba, B.M., Valle, M. et al. (2017). Implementation of a human papillomavirus vaccination demonstration project in Malawi: successes and challenges. BMC Public Health17, https://doi.org/10.1186/s12889-017-4526-y

49. Musa, Sanjin. Skrijelj, Venesa. Kulo, Aida. Habersaat, Katrine Bach. Smjecanin, Mirsad. Primorac, Emilija. Becirovic, Darija. Jackson, Cath. (2020). Identifying barriers and drivers to vaccination: A qualitative interview study with health workers in the Federation of Bosnia and Herzegovina, Vaccine, Volume 38, Issue 8, 1906-1914, https://doi.org/10.1016/j.vaccine.2020.01.025.

50. Naimoli, J. (2008). Toward a grounded theory of why some immunisation programme in sub saharan Africa are more successful than others: a Descriptive and exploratory study of Six countries. Health Policy and Planning, 379-389.

51. Nichter M. (1995). Vaccinations in the Third World: a consideration of community demand. Soc Sci Med. 41:617-32. PMID:7502096 doi:10.1016/0277-9536(95)00034-5 Streefland P, C. A.-J. (1999). Patterns of vaccination acceptance. Soc Sci Med., $1705-16$.

52. Ntenda, PAM. Mwenyenkulu, ET. Putthanachote, N. Nkoka, O. Mhone, TG. Motsa, MPS. Tizifa, T. (2019). Predictors of uptake of newly introduced vaccines in Malawi - monovalent human rotavirus and pneumococcal conjugate vaccines: Evidence from the $2015-16$ Malawi demographic and health survey, Journal of Tropical Pediatrics. Volume 65, Issue 3, Pages 287-296, https://doi.org/10.1093/tropej/fmy050

53. Nyumba, Tobias O., Wilson, Kerrie., Derrick, Christina J. and Mukherje, Nibedita (2017). The use of focus group discussion methodology: Insights from two decades of application in conservation. Methods Ecol Evol; 9: 20-32. https://doi.org/10.1111/2041-210X.12860

54. Ogbuanu IU, Li AJ, Anya BM, Tamadji M, Chirwa G, Chiwaya KW, Djalal ME, Cheikh D, Machekanyanga Z, Okeibunor J, Sanderson C, Mihigo R. (2019). Can vaccination coverage be improved by reducing missed opportunities for vaccination? Findings from assessments in Chad and Malawi using the new WHO methodology. PLoS One. 14(1):e0210648. doi: 10.1371/journal.pone.0210648.

55. Ogochukwu1, T.N. Akabueze, Jude. Ezeome1, I.J. Aniebue, U.U. and Oranu, E.O. (2017). Vaccination against Human Papilloma Virus in Adolescent Girls: Mother's Knowledge, Attitude, Desire and Practice in Nigeria. J Infect Dis Preve Med. 5:1. D0I: 10.4172/2329-8731.1000151

56. Okeibunor JC, Ogbuanu I, Blanche A, Chiwaya K, Chirwa G, Machekanyanga Z, Mihigo R., Zawaira, F. (2018). Towards a Strategy for Reducing Missed Opportunities for Vaccination in Malawi: Implications of a Qualitative Health Facility Assessment. Immunol Sci. Suppl(7):46-54.

57. Okwo, B., \& Jean, M. (2015). Together we can close the immunization gap. Geneva: World Health Organisation (WHO).

58. Oyo-Ita A, Wiysonge CS, Oringanje C, Nwachukwu CE, Oduwole O, Meremikwu MM. (2016). Interventions for improving coverage of childhood immunisation in low- and middle-income countries. Cochrane Database Syst Rev. 7(7):CD008145. doi: 10.1002/14651858.CD008145.pub3. Bottom of Form

59. Petrelli, F. (2018). Vaccine hesitancy, a public health problem. Annali di igiene : medicina preventiva e di comunita, 86-103.

60. Perlman, S., Wamai, R.G., Bain, P.A., Welty, T., Welty, E., Ogembo, J.G. (2014). Knowledge

90912. and awareness of HPV vaccine and acceptability to vaccinate in sub-Saharan Africa: a systematic review. PLoS ONE, 9(3): e90912.

61. Ruijs, W.L., Hautvast, J.L., van der Velden, K. et al. (2011). Religious subgroups influencing vaccination coverage in the Dutch Bible belt: an ecological study. BMC Public Health 11, 102. https://doi.org/10.1186/1471-2458-11-102 
62. Santos, A.C.D., Silva, N.N.T., Carneiro, C.M. et al.(2020). Knowledge about cervical cancer and HPV immunization dropout rate among Brazilian adolescent girls and their guardians. BMC Public Health. 20: 301. https://doi.org/10.1186/s12889-020-8410-9

63. Streefland P, Chowdhury AM, Ramos-Jimenez P. Patterns of vaccination acceptance. Soc Sci Med. 1999;49(12):1705-16.

64. Vanderslott, S., Dadonaite, B., \& Roser, M. (2019). Vaccination. Our world in Data. Retrieved January 2, 2020, from https://ourworldindata.org/vaccination.

65. Vermandere, H., Naanyu, V., Mabeya, H., Vanden Broeck, D., Michielsen, K., Degomme, O.

13. (2014). Determinants of acceptance and subsequent uptake of the HPV vaccine in a cohort in Eldoret, Kenya. PLoS One, 9(10), e109353: 1-13.

66. Wiyeh AB, Cooper S, Nnaji CA, Wiysonge CS. (2018). Vaccine hesitancy 'outbreaks': using epidemiological modeling of the spread of ideas to understand the effects of vaccine related events on vaccine hesitancy. Expert Rev Vaccines. 17(12).

67. WHO (2019a). Immunization coverage. Geneva: World Health Organization.

68. WHO (2020b). https://www.who.int/news-room/events/detail/2020/04/24/default-calendar/world-immunization-week-2020. Retrieve Jan. $2,2021$.

69. WHO (2017). Global Health Observatory data, Causes of Child Mortality. Retrieved January 23, 2020, from World Health Organization: https://www.who.int/gho/child_health/mortality/causes/en/

70. World Bank Group, n.d. Sub-Sahara Africa Data. Retrieve Dec. 7, 2020 from https://data.worldbank.org/country/ZG.

71. WHO (2020a). Vaccine-preventable diseases: monitoring system. 2020 global summary. Coverage time series for Malawi. Data as of 12October-2020. Retrieved Dec. 11, 2020. https://apps.who.int/immunization_monitoring/globalsummary/coverages?c=MWI

72. WHO/Strategic Advisory Working Group (SAGE) on Vaccination Hesitancy Report, (2014). https://www.who.int/immunization/sage/meetings/2014/october/SAGE_working_group_revised_report_vaccine_hesitancy.pdf. Retrieved December 28, 2019.

73. WHO (2019b). Ten threats to Global Health in 2019. Retrieved October 17, 2019, from World Health Organization. https://www.who.int/emergencies/ten-threats-to-global-health-in- 2019

74. WHO/UNICEF Estimates of National Immunisation Coverage (WUENIC), (2019a). Global Immunisation Dataset

75. WHO/UNICEF Estimates of National Immunisation Coverage (WUENIC), (2019b). Progress and Challenges with Achieving Universal Immunization Coverage. Retrieved September 8, 2020, from World Health Organisation: https://www.who.int/news-room/details/12-082019-progress-and-challenges-with-achieving-universal-immunizaation-coverage

\section{Figures}




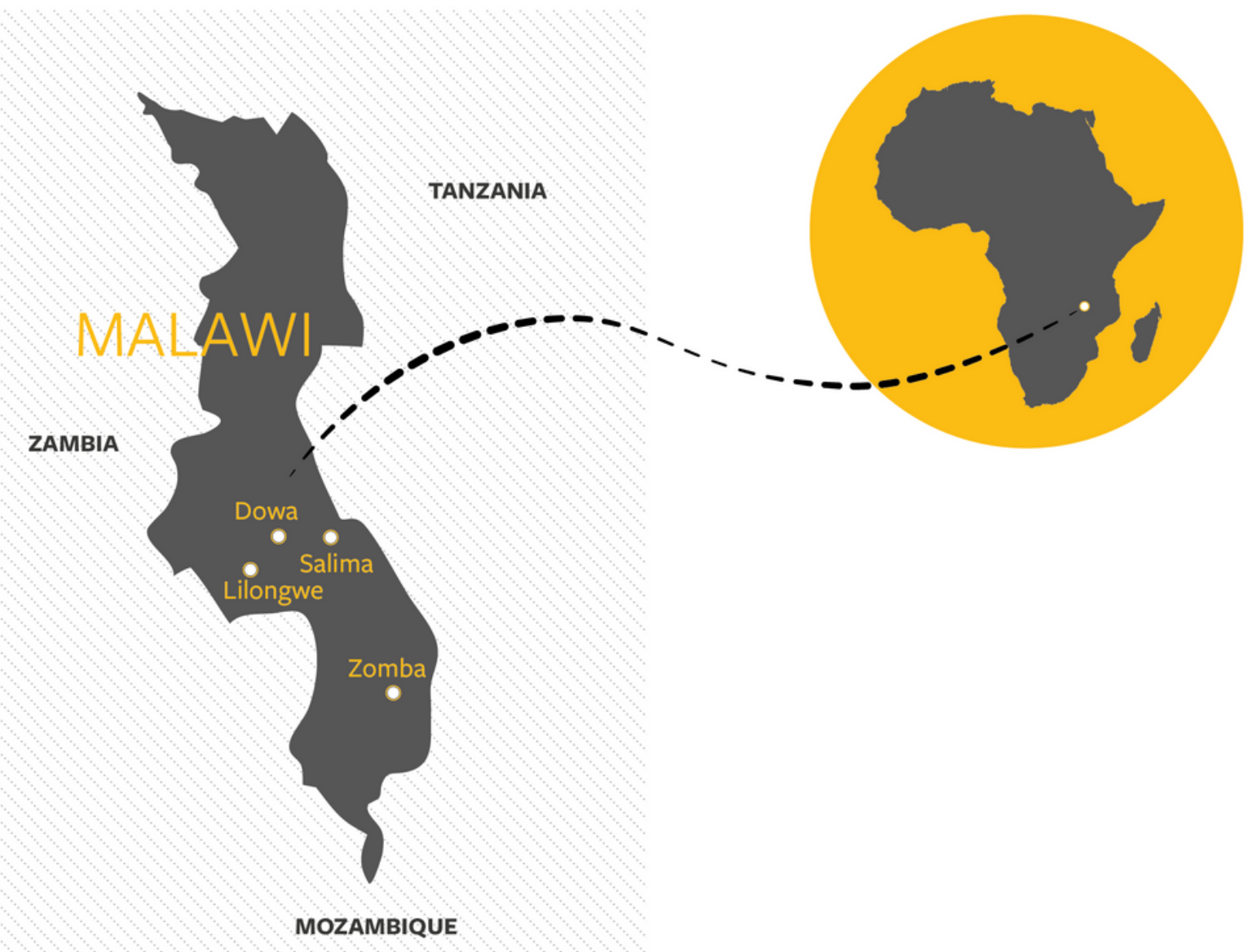

Figure 1

Map of study locations in Malawi Note: The designations employed and the presentation of the material on this map do not imply the expression of any opinion whatsoever on the part of Research Square concerning the legal status of any country, territory, city or area or of its authorities, or concerning the delimitation of its frontiers or boundaries. This map has been provided by the authors.

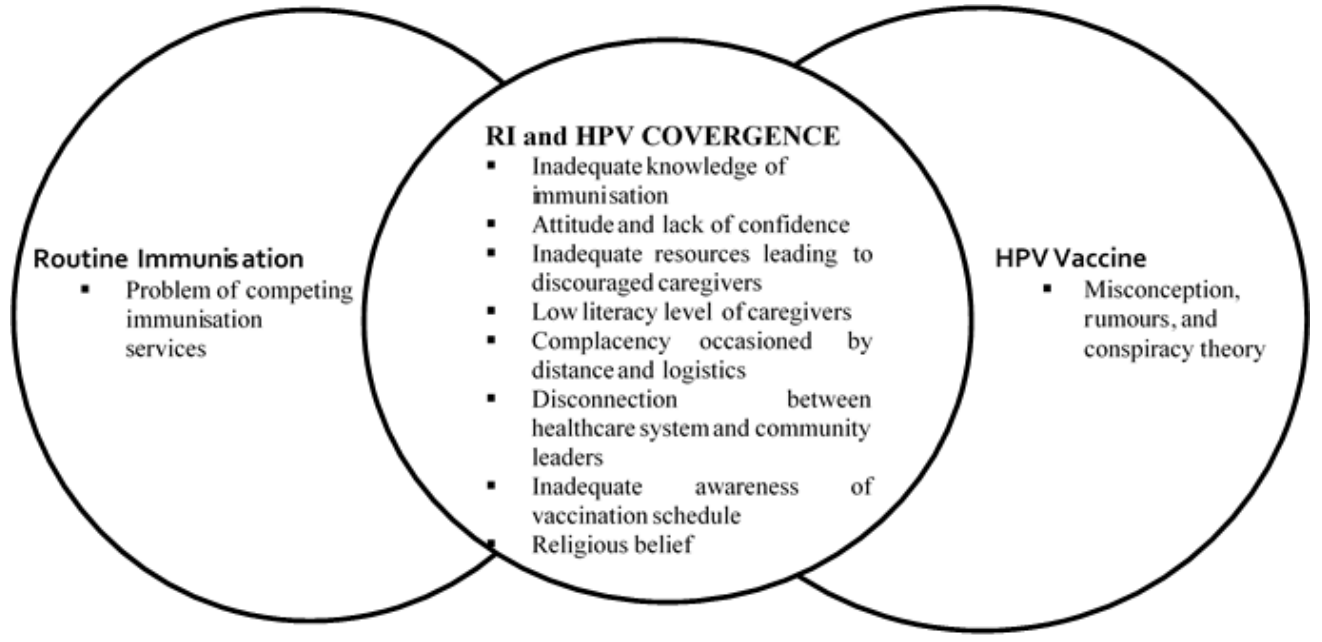

Figure 2 
Overview of identified drivers of vaccine hesitancy in Malawi for RI (left), HPV vaccination (right), and both (middle)

Page $14 / 14$ 